vaccination, differed significantly by Indigenous status, age group, Pap cytology status or area of residence.

Methods We used women attending for routine Pap smear, (April 2005-February 2008), as the sampling frame with 34 sites across Australia selected to include adequate numbers of Indigenous and remote-dwelling women. The final recruitment of 2620 (mean 33, range $15-66$ years) included $26 \%$ (684) indigenous. DNA extracts from Thin-Prep specimens were screened by HPV AMPLICOR (Roche) and in-house HPV PCR/ELISA, with positives genotyped by LINEAR ARRAY HPV genotyping test (Roche).

Results The overall prevalence of HPV infection was 39\% (95\% CI-36.8 to 40.6), with high risk (HR) HPV detected in $26 \%(95 \%$ CI-24.8 to 28.2). Single infections were detected in $17 \%(95 \%$ CI-15.8 to 18.7). While multiple infections were common overall at $22 \%$, there was no difference in proportion of multiple HPV carriage between indigenous and non-indigenous (58\% of HPV positive non-Indigenous women and $56 \%$ of HPV positive Indigenous women had multiple types detected). As with single infections, multiple type infections were less prevalent with increasing age. The six most common genotypes were-HPV 16 (8.3\%), 51 (5.1\%), 53 (4.7\%), 62 (4.3\%), 89 (3.9\%) and 52 (3.8\%). Age-specific HPV prevalence rates were similar for Indigenous and non-Indigenous women aged $\leq 30$, but higher for Indigenous women aged 31-40, particularly for non-vaccine targeted HR-HPV genotypes. By HPV clades for this age group, indigenous women were significantly more likely to have $\alpha 7 \mathrm{HPV}((45,39,59,68$ or 70 without 18$) \mathrm{OR}$ 1.9 (1.1 to 3.3) $\mathrm{p}=0.03$ ) or $\alpha 5$ group (HPV51, 26, 69, 82) with OR 2.1 (1.1 to 4.3$) \mathrm{p}=0.02)$. There was no significant association between Indigenous status and detection of HPV from the $\alpha 9$ clade (31, 33, 35, 52, 58, 67), with or without HPV 16. Overall, HR-HPV prevalence increased from $21 \%$ in women with normal cytology, to $81 \%$ in those with high-grade lesions/cancer.

Conclusions Cross-sectional prevalence of HR-HPV was high in Australian women, with vaccine preventable genotypes observed in $13 \%$ of all women ( $25 \%$ in $<25$ year olds). Vaccination should significantly reduce vaccine related HPV infection and disease in Australian women, irrespective of indigenous status or area of residence.

\section{P1-S1.54 HIGH-RISK HUMAN PAPILLOMA VIRUS (HPV) TYPES PREVALENCE IN 20-64-YEARS-OLD WOMEN; SLOVENIAN NATIONAL HPV PREVALENCE STUDY, 2010}

doi:10.1136/sextrans-2011-050108.54

${ }^{1}$ I Klavs, ${ }^{1} \mathrm{~V}$ Ucakar, ${ }^{2} \mathrm{M}$ Poljak. ${ }^{1}$ National Institute of Public Health, Ljubljana, Slovenia; ${ }^{2}$ Institute of Microbiology \& Immunology, Ljubljana, Slovenia

Background To estimate prevalence of 14 high-risk human papilloma virus (HPV) types among Slovenian women screened for cervical cancer.

Methods In 2010, we conducted a cross-sectional study in a convenience sample of 4469 women 20-64 years old, who were eligible for a preventive cytological examination of the cervical smear according to the criteria of the Slovenian National Cervical Cancer Screening Program, presented during the study period within a network of 16 outpatient gynaecology services with a nationally wide geographical coverage and consented to participate. We used three-step HPV genotyping strategy on cervical smear specimens positive with Digene Hybrid Capture 2 HPV DNA Test and/or Abbot Real Time High Risk HPV Test. Infection with high-risk HPV types was defined as the presence of one or more of the following $14 \mathrm{HPV}$ types-HPV16, HPV18, HPV31, HPV33, HPV35, HPV39, HPV45, HPV51, HPV52, HPV56, HPV58, HPV59, HPV66 and HPV68. The owerall prevalence of high-risk HPV types as well as individual highrisk HPV types was estimated with $95 \%$ CIs Statistical analyses were performed using the STATA package version 10.0.
Results Prevalence of cervical infection with any high-risk HPV type examined was $13.1 \%$ (CI-12.1 to 14.0$)$, prevalence of infection with HPV16 only was $3.5 \%(\mathrm{CI}-3.0$ to 4.1$)$ and prevalence of infection with HPV18 was 1.0 (CI-0.7 to 1.3). The corresponding age specific prevalence estimates decreased with age and were the highest among 20-24 years old women-26.0\% (CI 22.4 to 29.5), $9.2 \%$ (CI 6.8 to 11.5 ) and $1.9 \%$ (CI 0.8 to 3.0 ), respectively. Overall prevalence of infection with any high-risk HPV type examined was the lowest among participants without evidence of cervical disease $10.8 \%$ (CI 9.9 to 11.8 ) and increased with the severity of cervical disease to $72.5 \%$ (CI 61.7 to 83.3 ) in women with low grade squamous intraepithelial lesion (LSIL) and $83.7 \%$ (CI 72.2 to 95.2) in women with high grade squamous intraepithelial lesion (HSIL). Corresponding HPV16 prevalence estimates were 2.5\% (CI 2.0 to 3.0), 26.1 (CI 15.5 to 36.7 ) and $41.9 \%$ (CI 26.5 to 57.2 ), and corresponding HPV18 prevalence estimates were $0.9 \%$ (CI 0.6 to 1.2 ), $7.3 \%$ (CI 1.0 to 13.5 ) and $7.0 \%$ (CI 0.0 to 14.9 ).

Conclusions Our results provide baseline high-risk HPV types prevalence estimates and will inform future monitoring of the impact of HPV vaccination program, including possible replacement of nonvaccine HPV types and design of effective cervical cancer screening strategies.

\section{P1-S1.55 HIGHER SEROPREVALENCE IS ASSOCIATED WITH HPV INFECTIONS OF MUCOSAL EPITHELIUM AND INFECTIONS AT MULTIPLE SITES IN MEN}

doi:10.1136/sextrans-2011-050108.55

${ }^{1} \mathrm{~B}$ Lu, ${ }^{2} \mathrm{R}$ Viscidi, ${ }^{3} Y \mathrm{Wu},{ }^{1} \mathrm{~A}$ Nyitray, ${ }^{4} \mathrm{~L}$ Villa, ${ }^{5} \mathrm{E}$ Lazcano-Ponce, ${ }^{6} \mathrm{R}$ J Carvalho Silva, ${ }^{4} \mathrm{M}$ L Baggio, ${ }^{5} \mathrm{M}$ Quiterio, ${ }^{5} \mathrm{~J}$ Salmerón, ${ }^{1} \mathrm{D}$ Smith, ${ }^{1} \mathrm{M}$ Abrahamsen, ${ }^{1} \mathrm{M}$ Papenfuss, ${ }^{1}$ A Giuliano. ${ }^{1} \mathrm{H}$. Lee Moffitt Cancer Center and Research Institute, Tampa, USA; ${ }^{2}$ School of Medicine, Johns Hopkins University, Baltimore, USA; ${ }^{3}$ College of Public Health, University of South Florida, Tampa, USA; ${ }^{4}$ Ludwig Institute for Cancer Research, São Paulo, Brazil; 5 Instituto Nacional de Salud Pública, Cuernavaca, Mexico; ${ }^{6}$ Centre for Reference and Training in STD/AIDS, São Paulo, Brazil

Background Previously we observed elevated HPV seroprevalence in men who had sex with men (MSM) and men who had sex with men and women (MSMW) compared to men who had sex with women (MSW). We hypothesise that the higher seroprevalence is associated with HPV infection at multiple anatomic sites, and the presence of mucosal epithelium infection as opposed to keratinised epithelium infection. We assessed associations between HPV seropositivity and prevalent HPV infection at anal canal and external genitalia in men to test the hypothesis.

Methods Enrolment data for 1663 men, including 1474 MSW, 83 MSM and 106 MSMW, were analysed. HPV L1 VLP-based ELISA was used for serum antibody testing and Linear Array for HPV DNA testing. Associations were estimated for HPV 6 and 16, respectively, using logistic regression. MSM and MSMW were combined to form the group MSM.

Results Overall HPV 6 and 16 seroprevalence was 9.9\% and $14.1 \%$. HPV 6 and 16 DNA was present in $2.2 \%$ and $2.7 \%$ of anal samples, and $6.4 \%$ and $7.3 \%$ of genital samples. Seroprevalence of HPV 6 and 16 was significantly higher in MSM compared to MSW (32.8 vs $6.9 \%$; 34.4 vs $11.5 \%$ ). Similarly, HPV 6 and 16 DNA was more frequently detected in anal samples (9.5 vs $1.2 \%$; 9.0 vs $1.8 \%$ ) and genital samples (7.4 vs $6.2 \%$; 9.0 vs $7.1 \%$ ) of MSM than MSW. Men with simultaneous anal and genital HPV infection (AOR-9.20 and 6.79) or anal HPV infection alone (AOR-19.93 and 3.19) were significantly more likely to be HPV 6 and 16 seropositive. There was no association of seropositivity with prevalent genital HPV 6 or 16 infection. Strong positive associations were detected in MSW and MSM who were anal HPV 6 positive, regardless of coinfection with genital HPV 6. In contrast, no association was observed among MSW with anal HPV 16, with or without genital coinfection. 
However, significant associations were observed in MSM with anal HPV 16 (with coinfection-AOR, 10.94, 95\% CI, 1.18 to 101.68 ; without-AOR, 4.96, 95\% CI, 1.40 to 17.57 ).

Conclusion We found type-specific associations of HPV 6 and 16 seropositivity with prevalent anal HPV infection, but not with prevalent genital HPV infection alone. Anal HPV 6 infection was associated with seropositivity in both MSW and MSM, while anal HPV 16 infection was only associated with seropositivity in MSM. Our data suggest that, in men, anal HPV infection may be more efficient than genital HPV infection in inducing immune responses. This may have relevance for protective immunity or the lack thereof, conferred by natural infection.

\section{Epidemiology poster session 1: STI trends: Mycoplasma genitallium \\ P1-S1.56 THE INCIDENCE OF MYCOPLASMA GENITALIUM IN A COHORT OF YOUNG AUSTRALIAN WOMEN}

doi:10.1136/sextrans-2011-050108.56

\begin{abstract}
${ }^{1} \mathrm{~J}$ Walker, ${ }^{1} \mathrm{C}$ Fairley, ${ }^{2} \mathrm{C}$ Bradshaw, ${ }^{3} \mathrm{~S}$ Tabrizi, ${ }^{2} \mathrm{M}$ Chen, ${ }^{3} \mathrm{~J}$ Twin, ${ }^{3} \mathrm{~N}$ Taylor, ${ }^{4} \mathrm{~B}$ Donovan,
${ }^{4} \mathrm{~J}$ Kaldor, ${ }^{1} \mathrm{~J}$ Hocking. ${ }^{1}$ University of Melbourne, Australia; ${ }^{2}$ Melbourne Sexual Health Centre, Carlton, Australia; ${ }^{3}$ The Royal Women's Hospital, Parkville, Australia; ${ }^{4}$ National Centre in HIV Epidemiology and Clinical Research, Coogee, Australia
\end{abstract}

Background Mycoplasma genitalium (Mg) is an emerging sexually transmitted infection that has been associated with serious upper genital tract infections in women such as cervicitis, pelvic inflammatory disease and endometritis. The burden of disease for $\mathrm{Mg}$ in Australia is unknown as there are no current population prevalence or incidence data.

Methods Women aged 16-25 years were recruited from sexual health clinics (SHC) and general practice clinics (GP) in SouthEastern Australia and consented to participate in a 12-month study providing vaginal swabs through the mail. Women were tested at 6-monthly intervals for chlamydia and $\mathrm{Mg}$.

Results Overall, 1116 women were recruited from 29 clinics; with $79 \%$ of women retained at the conclusion of the study. The prevalence of $\mathrm{Mg}$ at recruitment was $2.4 \%$ (95\% CI 1.5 to 3.3). Increased numbers of sexual partners was strongly associated with $\mathrm{Mg}$ (adjusted OR $[\mathrm{AOR}]=2.2 ; 95 \%$ CI 1.0 to 4.6), as was being recruited from $\mathrm{SHC}(\mathrm{AOR}=3.4 ; 95 \% \mathrm{CI} 1.5$ to 5.3$)$. $\mathrm{Mg}$ incidence was 1.2 per 100 women years ( $95 \%$ CI 0.7 to 2.1 ) and was associated with women recruited from SHC ( $\mathrm{HR}=4.9 ; 95 \% \mathrm{CI} 1.5$ to 16.3$)$ and having increased numbers of new sexual partners ( $\mathrm{HR}=5.7 ; 95 \%$ CI 1.4 to 23.1). We found a median organism load of $1.4 \times 103 / 5$ ? L, which was 100 times less than that found in chlamydia positive samples. We also found an azithromycin failure rate of 15\% (95\% CI 3.2 to 37.9).

Conclusion $\mathrm{Mg}$ is common in young Australian women, and consistent with international studies, $\mathrm{Mg}$ was less prevalent than chlamydia.

\section{P1-S1.57 EPIDEMIOLOGY OF MYCOPLASMA GENITALIUM AND GENITAL HIV-1 RNA - A LONGITUDINAL STUDY AMONG HIV-INFECTED ZIMBABWWEN WOMEN}

doi:10.1136/sextrans-2011-050108.57

\begin{abstract}
${ }^{1} \mathrm{~S} N$ Mavedzenge, ${ }^{2} \mathrm{E}$ E Müller, ${ }^{2} \mathrm{D}$ A Lewis, ${ }^{3} \mathrm{~T}$ Chipato, ${ }^{4} \mathrm{C}$ Morrison, ${ }^{1} \mathrm{H}$ A Weiss. ${ }^{1}$ London School of Hygiene \& Tropical Medicine, London, UK; ${ }^{2}$ Sexually Transmitted Infections Reference Centre, Johannesburg, South Africa; ${ }^{3}$ University of Zimbabwe College of Health Science, Harare, Zimbabwe; ${ }^{4}$ Family Health International, Research Triangle Park, USA
\end{abstract}

Background Mycoplasma genitalium (MG) is an emerging STI associated with reproductive tract syndromes in men and women, and with HIV in cross-sectional studies. MG is common in HIV-infected women, but there have been no longitudinal studies of $M G$ and genital HIV RNA among HIV-infected women.

Methods The study is nested in a cohort of 131 HIV-infected, ARTnaïve Zimbabwean women aged 19-37 years. Real-time PCR was used to test for presence and quantity of MG DNA in 420 stored cervical samples (1-4 visits per woman). Genital and plasma HIV viral load, CD4 count and presence of other STI and reproductive tract infections were collected at each visit, together with clinical and behavioural data. Logistic and linear random-effects models were used to analyse i) factors associated with detection of MG, and ii) the association of detection and quantity of MG with detection and quantity of genital HIV RNA.

Results MG was detected at 44/420 (10.5\%) visits, with a median bacterial load of 1497 copies/ml (range <300-3240 000 copies/ml). MG was twice as prevalent as $N$ gonorrhoeae $(5.0 \%)$ or $C$ trachomatis (4.8\%). Of the 33 women with MG detected at least once, six were infected at $\geq 2$ consecutive visits, persisting for up to 43 weeks. In multivariable analyses, MG was independently associated with bacterial vaginosis $(\mathrm{OR}=2.24,95 \%$ CI 1.03 to 4.85), HSV2 $(\mathrm{OR}=8.56,95 \% \mathrm{CI} 0.99$ to 74.24$)$ and younger age ( $\mathrm{OR}=2.92,95 \%$ CI 1.10 to 7.76$)$. Cleaning inside the vagina was protective against MG infection ( $\mathrm{OR}=0.33,95 \%$ CI 0.15 to 0.71 ). Genital HIV RNA was detected at $237 / 397$ (59.7\%) visits, with a mean viral load of $5.14 \log 10$ copies/ml. MG was independently associated with detection of genital HIV RNA (OR=2.73, 95\% CI to $1.02-7.33$ ) after adjusting for confounders including plasma viral load, CD4 count, HSV2, and $N$ gonorrhoeae. Higher MG bacterial load was weakly associated with detection of genital HIV RNA (OR=1.75, $95 \%$ CI 0.96 to 3.19 ) but there was little association with quantity of HIV RNA.

Conclusions This cohort study confirms previous cross-sectional results showing an association of genital HIV DNA detection with $M G$ infection. Further research is needed to explore factors mediating this association, as MG was not associated with plasma viral load or measured markers of inflammation. The growing evidence for an association of MG with HIV genital shedding, and the high prevalence and persistence of MG infection, suggests that screening and treatment of MG may be warranted among HIV-positive women.

\section{Epidemiology poster session 2 : Population: Commercial sex worker \\ P1-S2.01 PREVALENCE OF HIV AND SEXUALLY TRANSMITTED INFECTIONS AMONG CLIENTS OF FEMALE SEX WORKERS IN KARNATAKA, SOUTH INDIA}

doi:10.1136/sextrans-2011-050108.58

${ }^{1} \mathrm{~S}$ Shaw, ${ }^{2} \mathrm{~K}$ Deering, ${ }^{1} \mathrm{~S}$ Reza-Paul, ${ }^{3} \mathrm{~S}$ Isac, ${ }^{3} \mathrm{~B}$ Ramesh, ${ }^{3} \mathrm{R}$ Washington, ${ }^{1} \mathrm{~S}$ Moses, ${ }^{1} \mathrm{~J}$ Blanchard. 'University of Manitoba, Winnipeg, Canada; ${ }^{2}$ University of British Columbia, Canada; ${ }^{3}$ Karnataka Health Promotion Trust, Bangalore, India

Introduction Several studies have demonstrated the importance of commercial sex work in the transmission of HIV and other sexually transmitted infections (STIs) in India. Clients of female sex workers (FSWs) are thought to be an important bridging population for HIV and STIs. However, there is little information on basic characteristics of sex work clients. This study sought to describe the prevalence of HIV and other STIs, as well as examine the determinants of these pathogens, among a sample of clients in south India

Methods Data were from a cross-sectional biological and behavioural survey of FSW clients from six districts in Karnataka State, India. The prevalence of HIV, syphilis, herpes simplex virus type 2 (HSV-2), chlamydia (CT) and gonorrhoea (GC) among clients was 